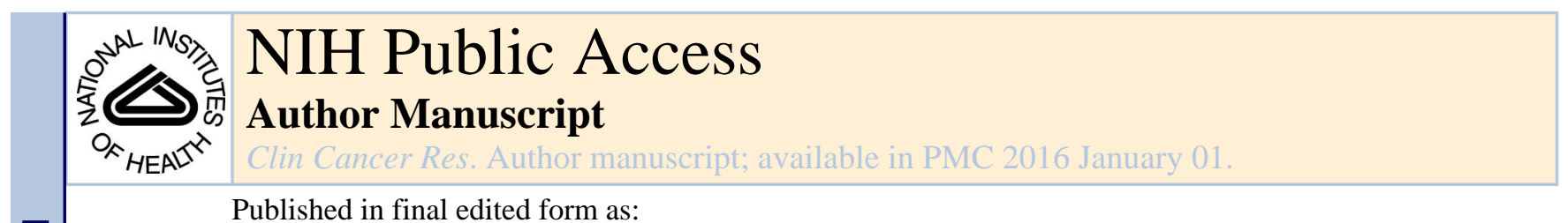

Published in final edited form as:

Clin Cancer Res. 2015 January 1; 21(1): 30-38. doi:10.1158/1078-0432.CCR-14-1716.

\title{
Tadalafil augments tumor specific immunity in patients with head and neck squamous cell carcinoma
}

\author{
Joseph A Califano ${ }^{*}{ }^{1,2,4}$, Zubair Khan ${ }^{1}$, Kimberly A. Noonan², Lakshmi Rudraraju ${ }^{2}$, Zhe \\ Zhang $^{3}$, Hao Wang ${ }^{3}$, Steven Goodman ${ }^{3}$, Christine G. Gourin ${ }^{1}$, Patrick K. Ha ${ }^{1,4}$, Carole \\ Fakhry $^{1,4}$, John Saunders ${ }^{4}$, Marshall Levine ${ }^{2,5}$, Mei Tang ${ }^{5}$, Geoffrey Neuner ${ }^{5}$, Jeremy D. \\ Richmon ${ }^{1}$, Ray Blanco ${ }^{4}$, Nishant Agrawal ${ }^{1}$, Wayne M. Koch ${ }^{1}$, Shanthi Marur ${ }^{2}$, Donald T. \\ Weed $^{6}$, Paolo Serafini ${ }^{7}$, and Ivan Borrello ${ }^{*}, 2$
}

${ }^{1}$ Department of Otolaryngology-Head and Neck Surgery, Johns Hopkins Medical Institutions, Baltimore, Maryland ${ }^{2}$ Oncology, Johns Hopkins Medical Institutions, Baltimore, Maryland ${ }^{3}$ Division of Biostatistics and Bioinformatics, Sidney Kimmel Comprehensive Cancer Center, Johns Hopkins Medical Institutions, Baltimore, Maryland ${ }^{4}$ Milton J Dance Head and Neck Center, Greater Baltimore Medical Center, Baltimore, Maryland ${ }^{5}$ Oncology Department, Greater Baltimore Medical Center, Baltimore, Maryland ${ }^{6}$ Department of Otolaryngology, University of Miami Miller School of Medicine, Miami, Florida ${ }^{7}$ Department of Microbiology and Immunology, University of Miami Miller School of Medicine, Miami, Florida

\section{Abstract}

Purpose-To determine if phosphodiesterase 5 (PDE5) inhibitors can augment immune function in head and neck cancer patients through inhibition of myeloid derived suppressor cells (MDSCs).

Experimental Design-We performed a randomized, prospective, double blinded, placebo controlled, phase II clinical trial to determine the in vivo effects of systemic PDE5 inhibition on immune function in head and neck squamous cell carcinoma (HNSCC) patients.

\begin{abstract}
Results-Tadalafil augmented immune response, increasing ex vivo $\mathrm{T}$ cell expansion to a mean 2.4 fold increase compared to 1.1 fold in control patients $(\mathrm{P}=0.01)$, reducing peripheral MDSC numbers to mean 0.81 fold change compared to a 1.26 fold change in control patients $(\mathrm{P}=0.001)$, and increasing general immunity as measured by delayed type hypersensitivity response $(\mathrm{P}=0.002)$. Tumor specific immunity in response to HNSCC tumor lysate was augmented in tadalafil treated patients $(\mathrm{P}=0.04)$.
\end{abstract}

Conclusions-These findings demonstrate that tadalafil augments general and tumor-specific immunity in HNSCC patients and has therapeutic potential in HNSCC. Evasion of immune surveillance and suppression of systemic and tumor specific immunity is a significant feature of head and neck cancer development. This study demonstrates that a PDE5 inhibitor, tadalafil, can

\footnotetext{
Address reprint requests to Dr. Califano and Dr. Borrello at the Department of Otolaryngology-Head and Neck Surgery and Oncology, Johns Hopkins Medical Institutions, 1550 Orleans Street, Room 5N.04, Baltimore, Maryland 21231, USA or at jcalifa@jhmi.edu or iborrell@jhmi.edu.

These authors contributed equally to this manuscript

Disclosures: PS and IB are named inventors on a patent owned by JHU regarding the use of PDE5 inhibitors as immune-modulators.
} 
reverse tumor specific immune suppression in head and neck cancer patients, with potential for therapeutic application.

\section{Background}

Head and neck squamous cell carcinoma (HNSCC) affects over 50,000 individuals annually in the United States.(1) Immune response is of critical importance in malignant transformation for a variety of solid tumors.(2) HNSCC patients demonstrate significant impairment in immune recognition of tumor cells, and evasion from immune response is a significant factor in HNSCC carcinogenesis. (3)

In vitro evidence suggests that immune-mediated tumor cell killing occurs through mechanisms distinct from chemotherapy-mediated killing and is a significant component of the therapeutic response.(4) In a study of colorectal cancer, characteristics of the immune infiltrate were more predictive of clinical outcomes than the classic TNM staging.(5) In ovarian cancer 5 year overall survival was $38.7 \%$ for patients with an intratumoral $\mathrm{T}$ cell infiltrate compared to $4.5 \%$ in those without detectable intratumoral $\mathrm{T}$ cells.(6) In addition, the presence of intratumoral regulatory $\mathrm{T}$ cells was associated with reduced survival in ovarian cancer.(7) There is a high rate of anergy for patients with HNSCC, with PPD nonresponsiveness in 50\% of patients, and anergy has been associated with poor survival. (8-17)

The immune system of cancer-bearing hosts has developed a complex network ultimately leading to the development of tumor-induced tolerance that includes regulatory $\mathrm{T}$ cells (Tregs), natural killer T cells (NKT), tumor associated macrophages (TAMs), indoleamine 2,3-dioxygenase (IDO) and myeloid derived suppressor cells (MDSCs).(18) However, MDSCs play an increasingly critical link between the innate and adaptive immune system through their ability to influence the fate of Tregs in antigen specific $\mathrm{T}$ cell tolerance.(19-21) MDSCs are induced by tumor-derived GM-CSF, VEGF and IL-6 and ultimately lead to T cell immune dysfunction through mechanisms including the production of arginase-1 (Arg1) and inducible nitric oxide synthase (NOS2). These two pathways lead to T cell dysfunction through the down-regulation of the $\zeta$-chain of the $\mathrm{T}$ cell receptor and nitrosylation of the tyrosines on the TCR of CD8 cells. $(22,23)$ Studies of MDSC-mediated immune suppression show that MDSCs exploit the metabolism of L-arginine (L-Arg) to render lymphocytes unresponsive to antigen stimulation $(24,25)$. NO-mediated suppression of T-cell activation is modulated by IL2 receptor signalling pathways and by direct pro-apoptotic effects.(26, 27) The ability of NOS inhibitors to reverse MDSC-induced immunosuppression both in vivo and in vitro confirms the immunoregulatory role of NO.

Functional elimination of MDSCs can overcome the immunosuppressive state,(28) and phosphodiesterase 5 (PDE5) inhibitors block both nitric oxide as well as arginase 1 production and restore tumor specific T cell function (Fig 1).(29) In vitro activation of peripheral T cells from HNSCC patients is increased with short acting PDE5 inhibitors.(29)

We hypothesized that PDE5 blockade may reverse tumor associated immune suppression in HNSCC patients and designed a randomized, placebo controlled, phase II biomarker endpoint trial in HNSCC patients to define the effects of an oral PDE5 blocking agent on peripheral $\mathrm{T}$ cell activation, global immune status, and MDSC function. We employed 
tadalafil, due to a favorable toxicity profile and the ability of this drug to provide long acting PDE5 blockade with once daily dosing.

\section{Methods}

Trial Design: We performed a double blinded, randomized two-arm placebo controlled study in which patients with biopsy proven head and neck squamous cell carcinoma were randomized to tadalafil $20 \mathrm{mg}$ once a day ( $20 \mathrm{mg}$ daily) for at least 10 days prior to their definitive therapy or to placebo prior to definitive therapy in a 5:3 ratio (Figure 1, online only). To define general systemic immunity, delayed type hypersensitivity (DTH) to Candida was determined prior to initiation of therapy and two days prior to the end of therapy. Induration and erythema was assessed at 48 hours. This clinical trial was approved by the Johns Hopkins Medical Institutions review board (Clinicaltrials.gov identifier NCT00894413), and all participants gave written informed consent to participate in the trial. Exclusion criteria included presence of existing immune suppression, a full list of inclusion and exclusion criteria are listed in Supplementary Materials. The majority of patients did not undergo surgical resection and were treated with primary radiation therapy (Supplementary Materials).

U266B1 (U266) and NCI-H929 (H929) myeloma cell lines were purchased from the ATCC and passaged as directed. Briefly, U266 and H929 cultured in complete RMPI media (Life Technologies, Grand Island NY) in T75 flasks at a concentration of $1 \times 10^{6}$ cells $/ \mathrm{ml}$ and $5 \times 10^{5}$ cells $/ \mathrm{ml}$ (respectively) with media renewal 2-3 times weekly. Cells are passaged no more than 3 total passages before aliquots are made for storage in the vapor phase of $1 \mathrm{~N} 2$. Cells are characterized by staining with human CD38 and CD138 (BD Biosciences, San Jose, $\mathrm{CA}$ ) and mycoplasma testing is conducted in the lab on a regular basis utilizing the PCR based MyctoDtect from Greiner BioOne (Monroe, NC). Head and neck cell lines are mycoplasma free based on PCR based MycoDtect ${ }^{\mathrm{TM}}$ kit from GreinerBio--One North America, Inc. (Monroe, NC) Cell lines are authenticated at Johns Hopkins Genetics Research Core Facility using a Short Tandem Repeats Profile Report for Cell Line Authentication (A Promega PowerPlex 16HS kit was used to polymerase chain (PCR) amplify fifteen short tandem repeat (STR) loci plus a gender determining marker, Amelogenin. The PCR product was electrophoresed on an ABI Prism® 3730×1 Genetic Analyzer using a Internal Lane Standard 600. Data was analyzed using GeneMapper® v 4.0 software (Applied Biosystems). Cell lines were confirmed within 6 months of lysate preparation. To produce lysate cells are grown up in large cultures so that at least 50 $100 \times 10^{6}$ cells can be obtained. Cell pellets are made by centrifuging the cellular culture. The cell pellets are exposed to a series of a minimum of 8 freeze/thaw cycles in dry ice/ isopropanol bath and then sonicated to further sheer the cellular membrane. Protein concentration analysis is performed utilizing a BSA protein kit (Thermo-Fisher Scientific, Waltham, MA).

\section{Statistical analyses}

The study was powered for 40 patients, 25 to tadalafil and 15 to placebo, calculated to provide $81 \%$ power for the statistical detection of a 0.8 SD difference in post-pre change 
between the two groups at one-sided Type I error rate of 0.05 . The study was terminated as per protocol after 40 patients were randomized. Of these 40 patients, 32 patients completed the full course of treatment and had both evaluable baseline and day 10-14 immune assessments. For comparison of treated and control group demographic variables values were calculated using Fisher's exact tests for categorical variables and Wilcoxon rank sum tests for continuous variables.

Pre and post-treatment change in the immune response outcome was quantified as a fold change (post/pre) and data was log transformed to improve normality (Table 1). A Wilcoxon rank sum test was performed for the comparison of immune response endpoints between the two arms due to the skewed distributions. Because only increased immunity would warrant further study of the regimen, statistical tests for the immune response outcomes were onesided unless otherwise indicated and statistical significance was considered at $P<0.05$. A multivariable linear regression analysis was further performed adjusting for baseline clinical variables including pre-treatment values, tobacco exposure, site, HPV status, HH and clinical $\mathrm{T}$ stage. The primary analyses $(\mathrm{n}=32)$ included patients who were randomized and completed full course of treatment and had baseline and post-baseline (Day 10) assessments. These analyses were repeated in an intent to treat population including all patients who were randomized $(\mathrm{n}=40)$, where conservative approach of baseline-observation-carried-forward, i.e., assuming no change in response to tadalafil administration, was applied to impute missing outcome data.

45 patients were initially screened in the study, 5 were excluded at the time of screening due to the presence of distant metastases $(n=3)$, high serum calcium $(n=1)$ or a second cancer $(\mathrm{n}=1)$. Eight patients (20\%) withdrew after enrollment (of these, 4 voluntarily discontinued prior to starting therapy due to trial logistics or enrollment in a competing trial, 2 due to findings of distant metastases/other cancers during routine staging after randomization prior to starting therapy, and 2 due to adverse events while on study related to study drug side effects (back pain). Patients with oropharynx HNSCC without available HPV status were excluded from analyses of HPV status and outcomes $(n=3)$; the remaining patients from non-oropharyngeal sites were considered HPV negative. Patients were followed until completion of therapy, but survival and disease control endpoints were not included as trial endpoints.

Descriptions of immune assays, molecular assays, and tumor specific immune assays are included in supplementary materials. Analyses were carried out using SAS (version 9.3, SAS Institute, Cary, NC) and R statistical software (version 2.15.2).

\section{Results}

\section{PDE-5 Inhibition Inhibits Immunosuppressive Function}

Of the 40 enrolled and randomized patients, 34 started treatment with tadalafil or placebo, 2 of these withdrew prior to completion of treatment due to back pain, and 32 completed therapy. We analyzed the 32 randomized patients who completed therapy to determine the effects of tadalafil on immune function. The tadalafil treated group had a higher proportion of smokers and higher $\mathrm{T}$ stage primary tumors (Table 2). 
We have previously shown that PDE inhibition suppresses iNOS and arginase-1 (Arg-1) production thereby inhibiting the immunosuppressive function of MDSCs. (29) Inducible NOS (iNOS) and Arg-1 production was determined by quantitative RT-PCR in isolated CD15+ cells. This approach was chosen in light of the fact that CD15 isolation would capture all the CD14+ (putative monocytic MDSCs) as well as the CD15+ cells (putative granulocytic MDSCs) (Supplemental Figure 4). Both Arg-1 and iNOS activity was significantly decreased in tadalafil treated patients with Arg1 showing a mean 0.83 fold change $(\mathrm{P}=0.004)$ in tadalafil treated patients versus a 1.0 fold change in control patients (Figure 2). iNOS was significantly reduced in tadalafil treated patients with a mean 0.66 fold change $(\mathrm{P}=0.003)$ yet slightly increased in control patients 1.02 fold (Figure 2).

The MDSC phenotype in this study was defined as $\mathrm{CD} 14^{+}$or $\mathrm{CD} 15^{+} / \mathrm{CD} 33^{+} / \mathrm{HLA}-$

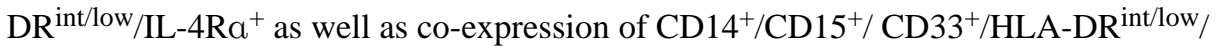
$\mathrm{IL}-4 \mathrm{Ra}^{+}$(See Supplementary Data for gating strategy). Inclusion of IL-4Ra is based on earlier studies showing the immunosuppressive function MDSCs expressing this phenotype. $(21,30)$ While no definitive phenotype has been identified for MDSCs, this phenotype was validated in the accompanying manuscript (Weed et al). We noted both a profound reduction in MDSC numbers with a mean 0.81 change in the treated cohort as well as a 1.28 fold increase in control patients $(\mathrm{P}<0.0001$, Figure 2$)$. These findings could suggest that PDE5 inhibition eliminates a positive autocrine feedback loop leading to either a shorter half-life of MDSCs or impaired de novo generation or that in its absence MDSC numbers can continue to increase. We previously showed that MDSC inhibition could reduce regulatory T cells (Tregs).(20) Our analysis demonstrated a statistically significant reduction in Tregs in the tadalafil-treated patients with a relative increase in the placebo group, Treg, mean placebo1.79, tadalafil $0.84,(\mathrm{p}=0.0006)$.

\section{PDE5 Inhibition Enhances Systemic Immunity}

Since MDSCs primarily suppress T cell function, we examined the effect of PDE5 inhibition. Peripheral blood mononuclear cells from both cohorts were stimulated with antiCD3/CD28 paramagnetic beads, and T cell expansion was determined by flow cytometry. Tadalafil treated patients showed a significant increase in $\mathrm{T}$ cell proliferation, with a mean 2.4 fold increase compared to a 1.1 fold increase in control patients $(\mathrm{P}=0.003)$ (Figure 3). Tadalafil increased $\mathrm{T}$ cell activation on both $\mathrm{CD} 4^{+} \mathrm{T}$ cells, mean 1.6 fold in tadalafil treated versus 1.3 fold in control treated patients $(\mathrm{p}=0.042)$, and CD8 T cells increased 1.4 fold versus no change $(\mathrm{p}=0.005)$ as measured by upregulation of CD69 expression on the T cell subpopulations (Table 3). Other immune response measures, including CD8 effector, CD 8 effector memory, CD8 naïve, and TCR Zeta populations did not show significant change (Supplementary Data).

To determine the impact of $\mathrm{T}$ cell modulation on global immunity, delayed type hypersensitivity (DTH) reactions were measured to Candida. Tadalafil treated patients showed a median 4.7 fold increase in DTH reactivity compared to a median 1.4 fold in the placebo treated patients $(\mathrm{p}=0.002)$ (Figure 3$)$ (Table 3$)$.

To further confirm the effect of tadalafil therapy, we performed a multivariate analysis to determine the potential confounding effect of clinical factors on immune measures. We 
examined potentially confounding factors between cohorts including $\mathrm{T}$ stage, site, tobacco exposure, and HPV status as this represents a major discriminating factor in HNSCC tumor biology. No immune differences were observed between HPV positive or negative patients (Supplementary Table 1, Supplementary Fig 2).

To confirm the robustness of this analysis, we examined an intent to treat analysis in all 40 patients enrolled in the trial regardless of completion of treatment. Univariate analysis showed that in the tadalafil intent to treat group there was a significant increase in $\mathrm{T}$ cell expansion $(\mathrm{P}=0.037)$, systemic response by DTH to Candida $(\mathrm{P}=0.032), \mathrm{CD} 4^{+} / \mathrm{CD} 69^{+}$cells $(\mathrm{P}=0.041)$, and $\mathrm{CD} 8^{+} / \mathrm{CD} 69^{+}$cells $(\mathrm{P}=0.014)$.

Using a multivariate model to control for tobacco exposure, site, HPV and T stage, the increase in $\mathrm{T}$ cell expansion in the tadalafil group remained significant $(\mathrm{p}=0.022)$. Similarly, the increase in CD8+ $\mathrm{T}$ cells in the tadalafil treated group remained significant $(\mathrm{p}=0.040)$ (Supplementary Data). We also found a tight correlation between changes in iNOS and Arg-1 expression and TCR zeta expression, MDSC decrease and enhanced immune response (Supplementary Table 5 and Supplementary Figure 3). The increase in systemic immunity and increase in CD4+ T cells did not retain significance on multivariate analysis.

We also examined the effect of dosage by examining body mass index (BMI) and body surface area (BSA) in relation to $\mathrm{T}$ cell activation and other immune response parameters in the tadalafil treated group. We did note a suggestion of decrease in immune effects as BMI and BSA increased potentially due to decreased tissue concentration of tadalafil, but this was not statistically significant. Increase in systemic immunity via DTH showed no variation in relation to BMI or BSA (Supplementary Data).

\section{PDE5 Inhibition Enhances Tumor Specific Immunity}

To determine the effect of PDE5 blockade on HNSCC specific immunity, we performed a sub-analysis on selected patients with available clinical material to examine tumor specific immunity to the mixture of HPV+ and HPV- HNSCC tumor lysate: three placebo treated and three tadalafil treated patients. The tadalafil treated patients demonstrated a significant increase in tumor specific immunity to HNSCC tumor lysate challenge after tadalafil therapy $(\mathrm{p}=0.04)$. Interestingly, this response was primarily $\mathrm{CD} 4$ restricted despite having observed a greater CD69 upregulation with tadalafil in the CD8 subset and greater proliferation of CD8 cells with PDE5 inhibition.(29)

\section{Discussion}

Despite recent advances in tumor targeted approaches, it is increasingly clear that the immune system not only is capable of directly imparting anti-tumor immunity but also plays a critical role in mediating tumor response to chemotherapy as well as potentiating the clinical efficacy of monoclonal antibody therapies such as cetuximab in HNSCC. $(31,32)$ These data reflect an increasing recognition of the complex role the immune system plays in mediating direct anti-tumor immunity and in potentiating the clinical efficacy of traditional cytotoxic therapies. MDSCs are increasingly recognized as playing a major 
immunosuppressive role in both the innate as well as adaptive immune responses and are major mediators of immune suppression in many solid tumors including HNSCC.(33)

Due to the critical role of MDSCs in mediating cancer-induced immune suppression, strategies aimed at abrogating their function have significant appeal. Recent approaches include promotion of myeloid differentiation, inhibition of their expansion as well as their elimination through the use of cytotoxic chemotherapy.(34) Our rationale for targeting PDE5 inhibition in this setting is to suppress MDSC function through inhibition of the two critical immunosuppressive pathways: iNOS and Arg-1.

We have previously hypothesized that the effect of PDE5 inhibition was on a destabilization of of NOS2 mRNA by reducing the ubiquitous mRNA binding protein, human antigen $\mathrm{R}$ (HuR), ultimately leading to a reduction in NO production (29) Ochoa and colleagues recently demonstrated how arginase activity was dependent upon cyclin D3 activity and how how stability of cyclin D3 mRNA was dependent upon HuR. (35) These latter findings establish a common link between PDE5 inhibition and abrogation of both the NO as well as Arg1 pathways.

These inhibitors are capable of inhibiting MDSC function, augmenting T cell function and exerting a measurable anti-tumor effect in murine models without exerting direct cytotoxic activity on either the MDSCs or tumor. (29) Utilizing an agent with no appreciable direct anti-tumor activity allows us to examine the direct role of MDSC inhibition on global and tumor-specific immunity. In this randomized study, we show that PDE5 inhibition suppresses iNOS and Arg-1 production, enhances global immune responsiveness, augments $\mathrm{T}$ cell function, and generates tumor specific immunity in HNSCC. These results highlight the critical role of MDSCs as mediators of immune suppressors in HNSCC and suggest the critical importance of inhibiting MDSC function in an effort to augment tumor specific immunity. This and the manuscript by Weed et al. is the first clinical study that demonstrates the ability of PDE5 inhibition to augment immune function in cancer patient, confirming an earlier observation.(36)

One unexpected finding was the observed decrease in MDSC numbers. PDE5 inhibitors are not known cytotoxic agents and the ex vivo addition of PDE5 inhibitors to MDSCs in culture failed to affect their survival (data not shown). This may be due to the short half-life of MDSCs and their endogenous requirement for cytokines produced by the MDSCs to stimulate cell growth in either an autocrine or paracrine manner. It is possible that by reducing iNOS and Arg-1 production, MDSCs alter the tumor microenvironment to either shorten their half-life or prevent the formation of other critical factors required for sustaining or generating additional MDSCs. The ability to adversely affect MDSC function, number, and possibly overall survival with a non-cytotoxic agent such as tadalafil is intriguing and could ultimately provide insight into the mechanisms of MDSC generation and persistence in cancer-bearing hosts. These data suggest that MDSCs may serve a critical role in immune response to HNSCC, that PDE5 inhibition is rapid and effective in abrogating MDSC function, and that eliminating MDSC function with PDE5 inhibition can restore both systemic and tumor specific immunity. 
Although we have shown that PDE inhibitors reduce NOS2 and ARG1, the full mechanisms underlying these effects remain to be elucidated. One putative mechanism involves the impact of these inhibitors on mRNA stability. cGMP destabilizes NOS2 mRNA by reducing the ubiquitous mRNA binding protein, human antigen $\mathrm{R}(\underline{45})$. As such, destabilization of NOS2 mRNA via PDE5 inhibition would abrogate NO-mediated immunosuppression more effectively than would competitive inhibition of NO itself. However, because ARG1 mRNA does not possess adenylate/ uridylate-rich elements and has not been described to be stabilized by human antigen R, other mechanisms are likely involved in PDE5-mediated down-regulation of ARG1. One possibility is that high levels of cGMP induced by PDE5 blockade reduce the cytosolic $\mathrm{Ca} 2+$ concentration, leading to a reduction of the calciumdependent protein kinase $\mathrm{C}$ activity that in turn prevents up-regulation of IL-4Ra. (37-39)

Interestingly, these effects on immune function were observed with only 10 days of treatment suggesting that inhibition of MDSC function can rapidly reverse immune suppression. A major mechanism involved in PDE5 inhibition is reduction in iNOS activity in the MDSCs. S-nitrosylation is a major protein regulator of numerous signaling pathways that significantly contribute to cell signaling.(40) Nitrosylation of the CD8 T cell receptor is a major mediator of MDSC-mediated $\mathrm{T}$ cell tolerance in cancer bearing hosts and nitrosylation of the chemokine CCL2 inhibited intratumoral T cell infiltration. $(22,41)$ Considering the reversibility of this mechanism, it is not surprising, albeit unexpected, that we observed such benefits with only 10 days of tadalafil administration. Of note, the observed increase in CD4+ T cells and increase in systemic immunity as determined by DTH response to Candida did not remain significant on multivariate analysis. This may be due to confounding effects of other variables, difficulties in quantitating systemic immunity via skin testing, or an inherent limitation given the relatively small sample size of this study,

The use of PDE5 inhibitors to augment tumor specific immunity has significant appeal. While widely used for erectile dysfunction, the use of these agents has been extended to pulmonary hypertension for which chronic administration of daily doses up to $40 \mathrm{mg}$ was associated with minimal toxicity. $(42,43)$ It should be noted that, to date, there are no studies showing any PDE5 inhibitor to have clinical anti-tumor activity as a single agent and this study was not designed to show anti-tumor efficacy. We believe the further clinical development of PDE5 inhibition in cancer will continue to focus on inhibition of MDSC function likely in combination with other immunotherapeutic modalities or more traditional cancer-based treatments. Ongoing clinical trials include the use of tadalafil to chronically modulate immune responses in combination with conventional therapy in HNSCC and to determine whether vaccine responses can be augmented with this approach.

\section{Supplementary Material}

Refer to Web version on PubMed Central for supplementary material.

\section{Acknowledgments}

We would like to thank Ervin Griffin for his assistance in processing and storing the samples. 
The manuscript/analysis of this article is based on a web database application provided by Research Information Technology Systems (RITS)

Funding: This study was supported by National Cancer Institute Grant 1R21CA135635 and National Cancer Institute/National Institute of Dental and Craniofacial Research Grant P50 CA19032.

\section{References}

1. Siegel R, Naishadham D, Jemal A. Cancer statistics, 2013. CA Cancer J Clin. 63:11-30. [PubMed: 23335087]

2. Coussens LM, Werb Z. Inflammation and cancer. Nature. 2002; 420:860-7. [PubMed: 12490959]

3. Kim MM, Califano JA. Molecular pathology of head-and-neck cancer. Int J Cancer. 2004; 112:54553. [PubMed: 15382034]

4. Ravi R, Fuchs EJ, Jain A, Pham V, Yoshimura K, Prouser T, et al. Resistance of cancers to immunologic cytotoxicity and adoptive immunotherapy via $\mathrm{X}$-linked inhibitor of apoptosis protein expression and coexisting defects in mitochondrial death signaling. Cancer Res. 2006; 66:1730-9. [PubMed: 16452233]

5. Galon J, Costes A, Sanchez-Cabo F, Kirilovsky A, Mlecnik B, Lagorce-Pages C, et al. Type, density, and location of immune cells within human colorectal tumors predict clinical outcome. Science. 2006; 313:1960-4. [PubMed: 17008531]

6. Zhang L, Conejo-Garcia JR, Katsaros D, Gimotty PA, Massobrio M, Regnani G, et al. Intratumoral T cells, recurrence, and survival in epithelial ovarian cancer. N Engl J Med. 2003; 348:203-13. [PubMed: 12529460]

7. Curiel TJ, Coukos G, Zou L, Alvarez X, Cheng P, Mottram P, et al. Specific recruitment of regulatory $\mathrm{T}$ cells in ovarian carcinoma fosters immune privilege and predicts reduced survival. Nat Med. 2004; 10:942-9. [PubMed: 15322536]

8. Pumhirun P, Wasuwat P. Anergy testing in patients with head and neck cancer. Asian Pac J Allergy Immunol. 2003; 21:189-92. [PubMed: 15032403]

9. Zighelboim J, Dorey F, Parker NH, Calcaterra T, Ward P, Fahey JL. Immunologic evaluation of patients with advanced head and neck cancer receiving weekly chemoimmunotherapy. Cancer. 1979; 44:117-23. [PubMed: 313240]

10. Wanebo HJ, Pinsky CM, Beattie EJ Jr, Oettgen HF. Immunocompetence testing in patients with one of the four common operable cancers--a review. Clin Bull. 1978; 8:15-22. [PubMed: 668106]

11. Kenefick TC. Delayed hypersensitivity skin test in head and neck cancer. J Laryngol Otol. 1976; 90:935-43. [PubMed: 993657]

12. Woods JE. The influence of immunologic responsiveness on head and neck cancer. Therapeutic implications. Plast Reconstr Surg. 1975; 56:77-80. [PubMed: 1144550]

13. Hazra TA, Parks LC, Inalsingh A, Peeples W. Delayed hypersensitivity to DNCB and survival following radiation therapy in patients with solid malignant tumors. Radiology. 1975; 115:429-30. [PubMed: 1144761]

14. Lundy J, Wanebo H, Pinsky C, Strong E, Oettgen H. Delayed hypersensitivity reactions in patients with squamous cell cancer of the head and neck. Am J Surg. 1974; 128:530-3. [PubMed: 4429642]

15. Eilber FR, Morton DL, Ketcham AS. Immunologic abnormalities in head and neck cancer. Am J Surg. 1974; 128:534-8. [PubMed: 4422512]

16. Mandel MA, Kiehn CL. The prognostic significance of delayed cutaneous reactivity in head and neck cancer patients. Plast Reconstr Surg. 1974; 53:72-6. [PubMed: 4588603]

17. Maisel RH, Ogura JH. Abnormal dinitrochlorobenzene skin sensitization: a prognostic sign of survival in head and neck squamous cell carcinoma. Laryngoscope. 1973; 83:2012-9. [PubMed: 4772104]

18. Rabinovich GA, Gabrilovich D, Sotomayor EM. Immunosuppressive strategies that are mediated by tumor cells. Annu Rev Immunol. 2007; 25:267-96. [PubMed: 17134371] 
19. Serafini P, Borrello I, Bronte V. Myeloid suppressor cells in cancer: recruitment, phenotype, properties, and mechanisms of immune suppression. Semin Cancer Biol. 2006; 16:53-65. [PubMed: 16168663]

20. Serafini P, Mgebroff S, Noonan K, Borrello I. Myeloid-derived suppressor cells promote crosstolerance in B-cell lymphoma by expanding regulatory T cells. Cancer Res. 2008; 68:5439-49. [PubMed: 18593947]

21. Walter S, Weinschenk T, Stenzl A, Zdrojowy R, Pluzanska A, Szczylik C, et al. Multipeptide immune response to cancer vaccine IMA901 after single-dose cyclophosphamide associates with longer patient survival. Nat Med. 18:1254-61. [PubMed: 22842478]

22. Nagaraj S, Gupta K, Pisarev V, Kinarsky L, Sherman S, Kang L, et al. Altered recognition of antigen is a mechanism of CD8+ T cell tolerance in cancer. Nat Med. 2007; 13:828-35. [PubMed: 17603493]

23. Rodriguez PC, Quiceno DG, Zabaleta J, Ortiz B, Zea AH, Piazuelo MB, et al. Arginase I production in the tumor microenvironment by mature myeloid cells inhibits T-cell receptor expression and antigen-specific T-cell responses. Cancer Res. 2004; 64:5839-49. [PubMed: 15313928]

24. Bogdan C. Nitric oxide and the immune response. Nat Immunol. 2001; 2:907-16. [PubMed: 11577346]

25. Wu G, Morris SM Jr. Arginine metabolism: nitric oxide and beyond. Biochem J. 1998; 336(Pt 1): 1-17. [PubMed: 9806879]

26. Pericle F, Kirken RA, Bronte V, Sconocchia G, DaSilva L, Segal DM. Immunocompromised tumor-bearing mice show a selective loss of STAT5a/b expression in T and B lymphocytes. J Immunol. 1997; 159:2580-5. [PubMed: 9300676]

27. Pericle F, Pinto LA, Hicks S, Kirken RA, Sconocchia G, Rusnak J, et al. HIV-1 infection induces a selective reduction in STAT5 protein expression. J Immunol. 1998; 160:28-31. [PubMed: 9551952]

28. Terabe M, Matsui S, Park JM, Mamura M, Noben-Trauth N, Donaldson DD, et al. Transforming growth factor-beta production and myeloid cells are an effector mechanism through which CD1drestricted $\mathrm{T}$ cells block cytotoxic $\mathrm{T}$ lymphocyte-mediated tumor immunosurveillance: abrogation prevents tumor recurrence. J Exp Med. 2003; 198:1741-52. [PubMed: 14657224]

29. Serafini P, Meckel K, Kelso M, Noonan K, Califano J, Koch W, et al. Phosphodiesterase-5 inhibition augments endogenous antitumor immunity by reducing myeloid-derived suppressor cell function. J Exp Med. 2006; 203:2691-702. [PubMed: 17101732]

30. Gallina G, Dolcetti L, Serafini P, De Santo C, Marigo I, Colombo MP, et al. Tumors induce a subset of inflammatory monocytes with immunosuppressive activity on CD8+ T cells. J Clin Invest. 2006; 116:2777-90. [PubMed: 17016559]

31. Apetoh L, Ghiringhelli F, Tesniere A, Obeid M, Ortiz C, Criollo A, et al. Toll-like receptor 4dependent contribution of the immune system to anticancer chemotherapy and radiotherapy. Nat Med. 2007; 13:1050-9. [PubMed: 17704786]

32. Srivastava RM, Lee SC, Andrade Filho PA, Lord CA, Jie HB, Davidson HC, et al. Cetuximabactivated natural killer and dendritic cells collaborate to trigger tumor antigen-specific T-cell immunity in head and neck cancer patients. Clin Cancer Res. 19:1858-72. [PubMed: 23444227]

33. Ostrand-Rosenberg S, Sinha P. Myeloid-derived suppressor cells: linking inflammation and cancer. J Immunol. 2009; 182:4499-506. [PubMed: 19342621]

34. Gabrilovich DI, Nagaraj S. Myeloid-derived suppressor cells as regulators of the immune system. Nat Rev Immunol. 2009; 9:162-74. [PubMed: 19197294]

35. Rodriguez PC, Hernandez CP, Morrow K, Sierra R, Zabaleta J, Wyczechowska DD, et al. Larginine deprivation regulates cyclin D3 mRNA stability in human T cells by controlling HuR expression. Journal of immunology. 2010; 185:5198-204.

36. Noonan K, Ghosh N, Rudraraju L, Marilyn Bui M, Borrello I. Targeting Immune Suppression with PDE-5 Inhibition in End-Stage Multiple Myeloma: A Case Study. Cancer Immunology Research. 2014; 2:725-731. [PubMed: 24878583]

37. Rotella DP. Phosphodiesterase 5 inhibitors: current status and potential applications. Nature reviews Drug discovery. 2002; 1:674-82. 
38. Vellenga E, Dokter W, Halie RM. Interleukin-4 and its receptor; modulating effects on immature and mature hematopoietic cells. Leukemia. 1993; 7:1131-41. [PubMed: 8350614]

39. Webb BL, Hirst SJ, Giembycz MA. Protein kinase C isoenzymes: a review of their structure, regulation and role in regulating airways smooth muscle tone and mitogenesis. British journal of pharmacology. 2000; 130:1433-52. [PubMed: 10928943]

40. Hess DT, Matsumoto A, Kim SO, Marshall HE, Stamler JS. Protein S-nitrosylation: purview and parameters. Nat Rev Mol Cell Biol. 2005; 6:150-66. [PubMed: 15688001]

41. Molon B, Ugel S, Del Pozzo F, Soldani C, Zilio S, Avella D, et al. Chemokine nitration prevents intratumoral infiltration of antigen-specific T cells. J Exp Med. 2011; 208:1949-62. [PubMed: 21930770]

42. Archer SL, Michelakis ED. Phosphodiesterase type 5 inhibitors for pulmonary arterial hypertension. N Engl J Med. 2009; 361:1864-71. [PubMed: 19890129]

43. Oudiz RJ, Brundage BH, Galie N, Ghofrani HA, Simonneau G, Botros FT, et al. Tadalafil for the treatment of pulmonary arterial hypertension: a double-blind 52-week uncontrolled extension study. J Am Coll Cardiol. 60:768-74. [PubMed: 22818063] 


\section{Translational Relevance}

HNSCC patients demonstrate significant impairment in immune recognition of tumor cells, and evasion from immune response is a significant factor in HNSCC carcinogenesis. These data demonstrate that tadalafil was effective in reversing suppression of immune recognition of head and neck cancer in patients taking daily tadalafil. Tadalafil was chosen due to a favorable toxicity profile and the ability of this drug to provide long acting effect with once daily dosing. Ongoing clinical trials include the use of tadalafil to chronically modulate immune responses in combination with conventional therapy in HNSCC and to determine whether vaccine responses can be augmented with this approach. 
a)
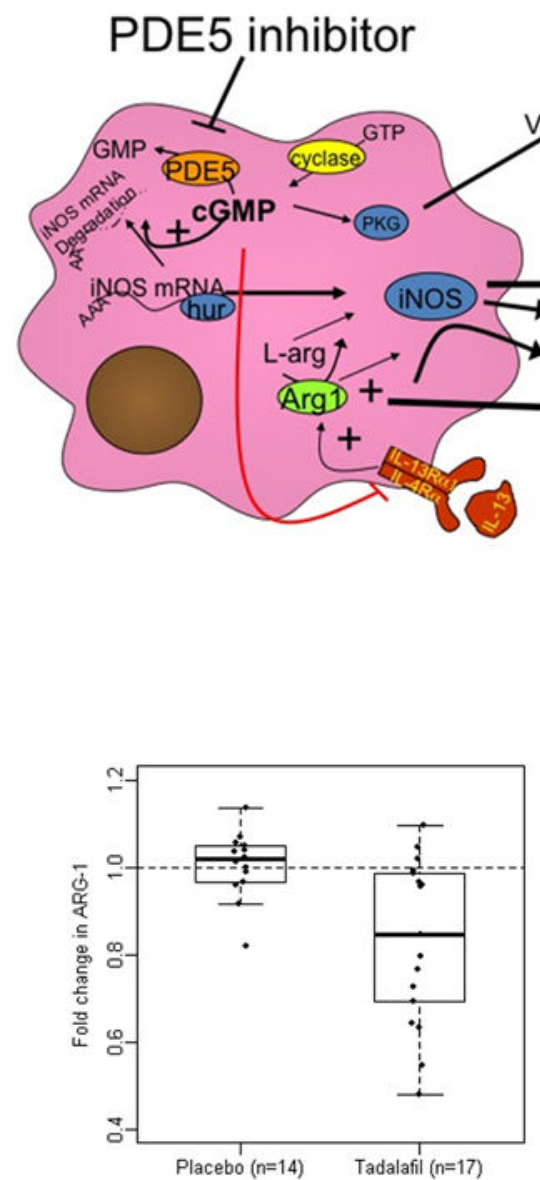

b)

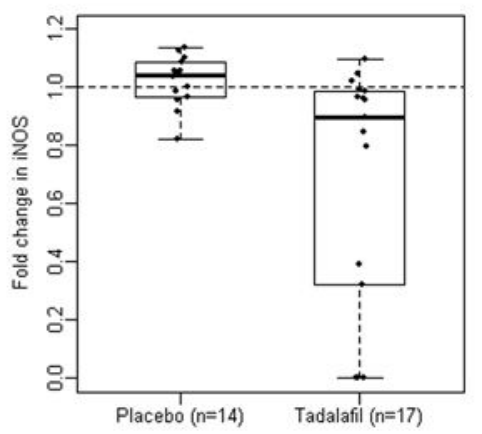

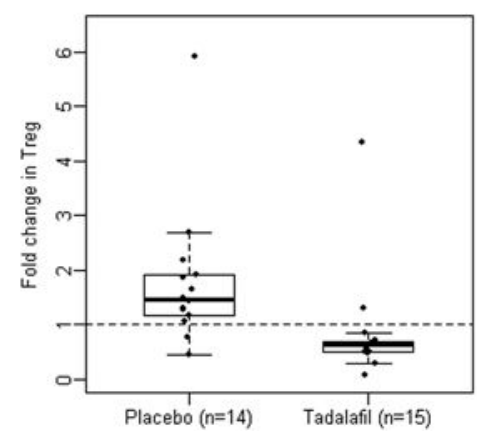

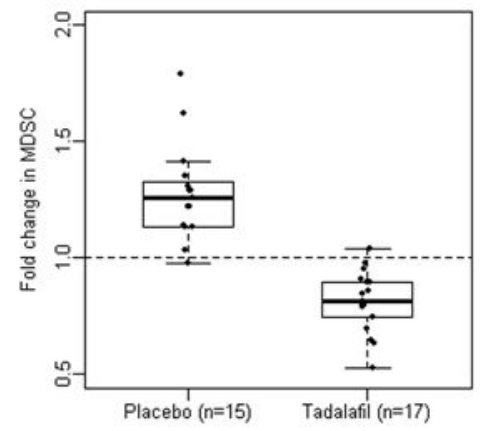

Figure 1.

A. Schematic Representation of PDE5 Blockade on MDSC Function: PDE inhibition increases cGMP which results in destabilization of the iNOS mRNA, reduced synthesis of iNOS and ultimately less production of NO. It is also able to down-regulate the expression of IL4Ra resulting in a reduction of arginase-1 expression. Taken together, these effects lead to a reversal of the immunosuppressive properties of MDSCs.

B. Tadalafil reduces MDSC function: Quantitative RT-PCR was performed on CD15 isolated peripheral blood cells. Tadalafil treatment resulted in: a decrease in Arg-1 
production. Mean fold change pre- vs post-treatment for placebo 1.0, tadalafil 0.83 $(\mathrm{p}=0.004)$; a decrease in iNOS, mean placebo 1.02, tadalafil 0.66. $(\mathrm{p}=0.003)$; a decrease in Treg, mean placebo1.79, tadalafil 0.84, $(\mathrm{p}=0.0006)$; and a decrease in MDSC, mean placebo 1.28 , tadalafil $0.81,(\mathrm{p}<0.0001)$. The length of the box is the inter-quartile range and represents the middle $50 \%$ of the data. The horizontal line inside the box shows the median. The lower and upper hinges of the box represent the 25th and 75th percentiles, respectively. The vertical dashed lines extend from the box to the upper and lower 1.5 inter-quartile values from the upper and lower hinges. The filled circles represent the actual values of the fold change where potential outliers are indicated by open circles. 

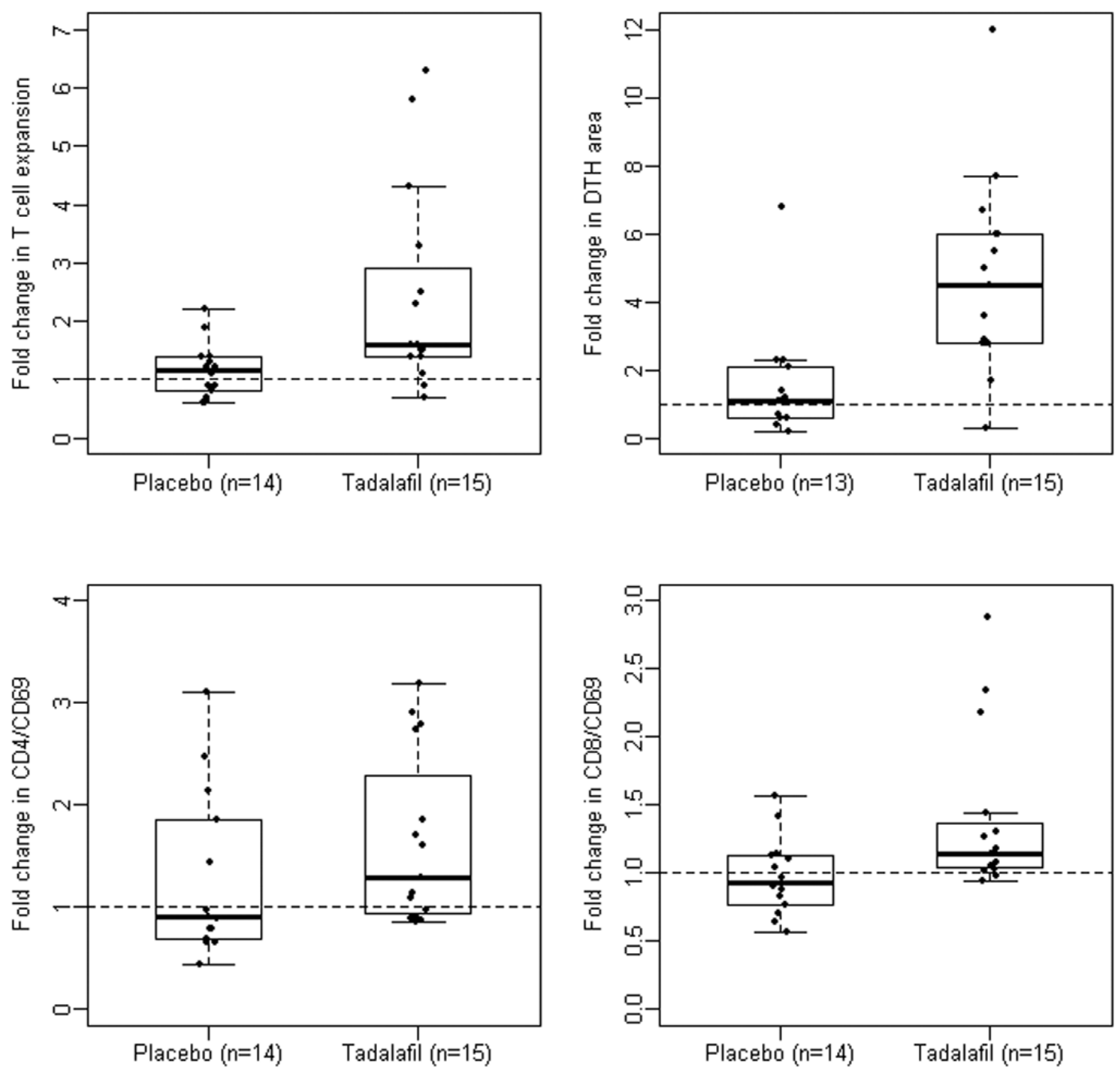

Figure 2. Tadalafil augments immune function

Fold increase in T cell expansion was determined following anti-CD3/CD28 stimulation.

Expansion was significantly greater in tadalafil-treated arm 2.4 fold vs placebo 1.1 fold $(\mathrm{p}=0.003)$. Systemic immune responsiveness as measured by fold change in area of induration from a DTH response to Candida with an increase in response in tadalafil treated patients $(\mathrm{p}=0.002)$, Change in activated T cells as assessed by CD69 expression: peripheral $\mathrm{CD}^{+}$cells, and peripheral $\mathrm{CD}^{+}$cells. Two outliers with a large fold change in DTH area suppressed, these are included in Supplementary Figure 5. Figures reflect data points as in Figure 2. 


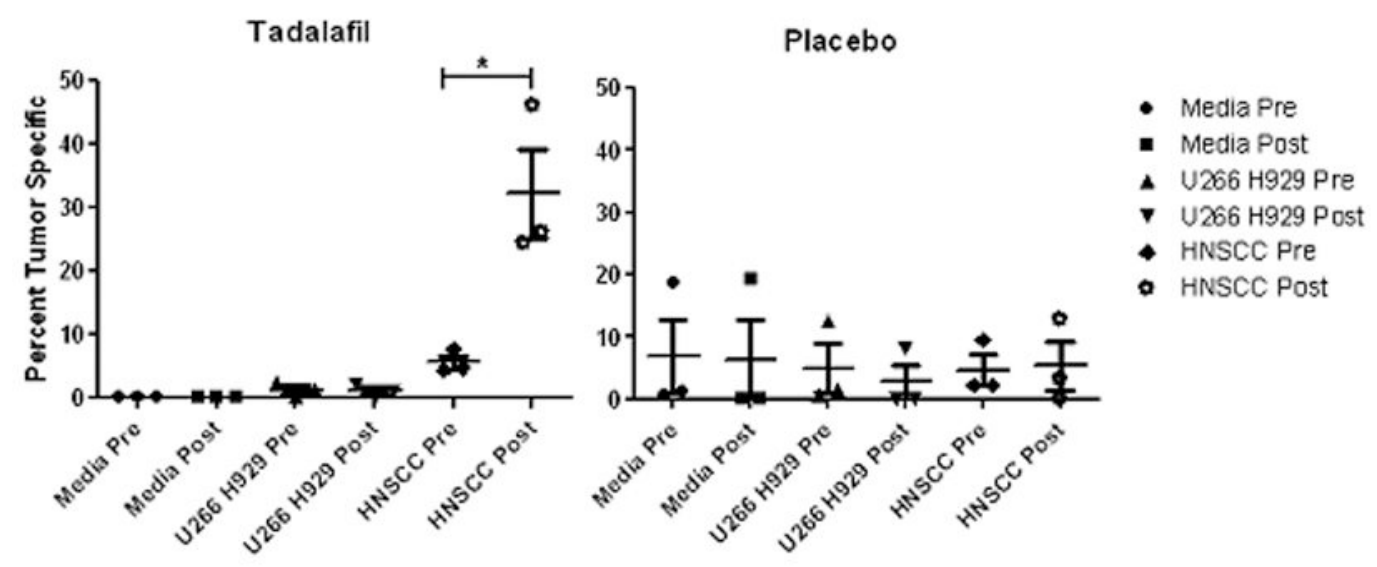

Figure 3. Tadalafil increases HNSCC specific immunity

CFSE-labeled T cells obtained pre- and post-treatment were added to dendritic cells pulsed with the indicated tumor lysate of either a mixture of HPV+ and HPV- HNSCC cell lines or myeloma cell lines (U266, H929). Tumor specificity was determined after a 7-day coculture by flow cytometry as CFSE ${ }^{\text {low } / ~ I F N ~} \gamma^{+}$CD3 cells. Shown is the change in tumor specificity in the $\mathrm{CD}^{+} \mathrm{T}$ cells in the placebo or tadalafil treated groups $(\mathrm{p}=0.04)$. No significant changes in tumor specificity were observed in the $\mathrm{CD} 8^{+} \mathrm{T}$ cells. 


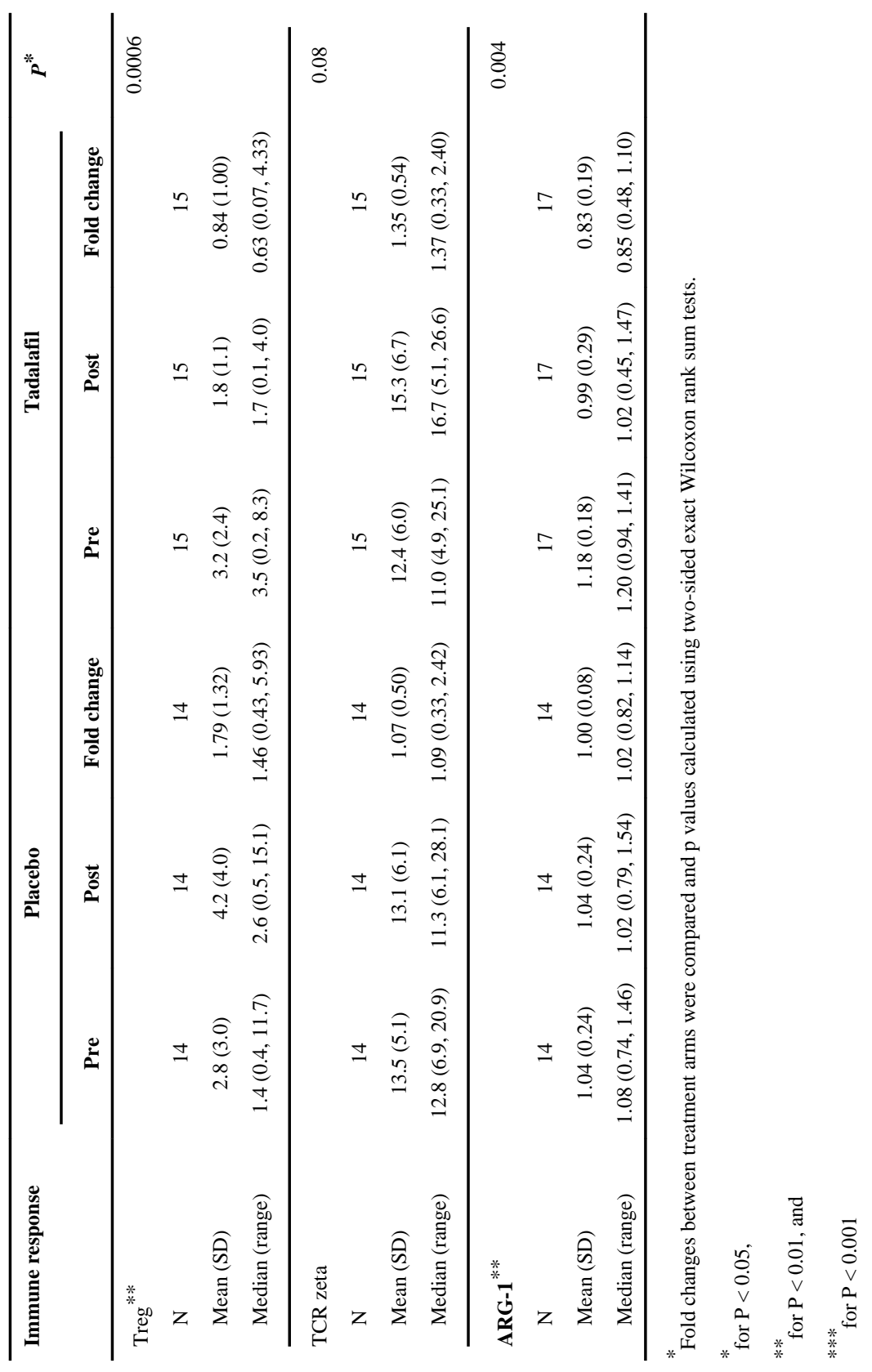


Table 2

\section{Baseline Patient Characteristics}

\begin{tabular}{|c|c|c|c|c|}
\hline Characteristics & $\operatorname{Total}(\mathrm{n}=32)$ & $\operatorname{Placebo}(n=15)$ & Tadalafil $(n=17)$ & $P^{*}$ \\
\hline Age (years) & & & & 0.34 \\
\hline Mean (SD) & $58(12)$ & $55(6)$ & $60(15)$ & \\
\hline Median (range) & $57(34-90)$ & $56(43-64)$ & $57(34-90)$ & \\
\hline Gender, $\mathrm{n}[\%]$ & & & & 0.59 \\
\hline Male & $29(91)$ & $13(87)$ & $16(94)$ & \\
\hline Female & $3(9)$ & $2(13)$ & $1(6)$ & \\
\hline Race, $\mathrm{n}[\%]$ & & & & $>0.99$ \\
\hline White & $27(84)$ & $13(87)$ & $14(82)$ & \\
\hline Black/Other & $5(16)$ & $2(13)$ & $3(18)$ & \\
\hline Tobacco exposure, $\mathrm{n}[\%]$ & & & & 0.023 \\
\hline Never & $10(31)$ & $8(53)$ & $2(12)$ & \\
\hline Ever & $21(66)$ & $7(47)$ & $14(82)$ & \\
\hline Missing & $1(3)$ & $0(0)$ & $1(6)$ & \\
\hline ETOH exposure, n [\%] & & & & 0.44 \\
\hline Never & $10(31)$ & $6(40)$ & $4(24)$ & \\
\hline Ever & $15(53)$ & $7(47)$ & $10(59)$ & \\
\hline Missing & $5(16)$ & $2(13)$ & $3(17)$ & \\
\hline Site, $n[\%]$ & & & & 0.063 \\
\hline Hypopharynx/Larynx & $7(22)$ & $1(7)$ & $6(35)$ & \\
\hline Oral cavity & $7(22)$ & $5(33)$ & $2(12)$ & \\
\hline Oropharynx & $15(47)$ & $9(60)$ & $6(35)$ & \\
\hline Unknown & $3(9)$ & $0(0)$ & $3(18)$ & \\
\hline Clinical T stage, $\mathrm{n}[\%]$ & & & & 0.029 \\
\hline $\mathrm{T} 0 / \mathrm{Tx} / \mathrm{T} 1 / \mathrm{T} 2$ & $18(56)$ & $12(80)$ & $6(35)$ & \\
\hline $\mathrm{T} 3 / \mathrm{T} 4$ & $13(41)$ & $3(20)$ & $10(59)$ & \\
\hline Missing & $1(3)$ & $0(0)$ & $1(6)$ & \\
\hline Clinical $\mathrm{N}$ stage, $\mathrm{n}[\%]$ & & & & $>0.99$ \\
\hline $\mathrm{N} 0 / \mathrm{N} 1$ & $14(44)$ & $7(47)$ & $7(41)$ & \\
\hline $\mathrm{N} 2 / \mathrm{N} 3$ & $17(53)$ & $8(53)$ & $9(53)$ & \\
\hline Missing & $1(3)$ & $0(0)$ & $1(6)$ & \\
\hline HPV status, n [\%] & & & & 0.27 \\
\hline Negative & $16(50)$ & $6(40)$ & $10(59)$ & \\
\hline Positive & $13(41)$ & $8(53)$ & $5(29)$ & \\
\hline Unknown & $3(9)$ & $1(7)$ & $2(12)$ & \\
\hline
\end{tabular}

Clin Cancer Res. Author manuscript; available in PMC 2016 January 01. 
* $\mathrm{P}$ values were calculated using two-sided exact Chi-square and Fisher's exact tests for categorical variables and exact Wilcoxon rank sum tests for continuous variables, with missing/unknown excluded. 


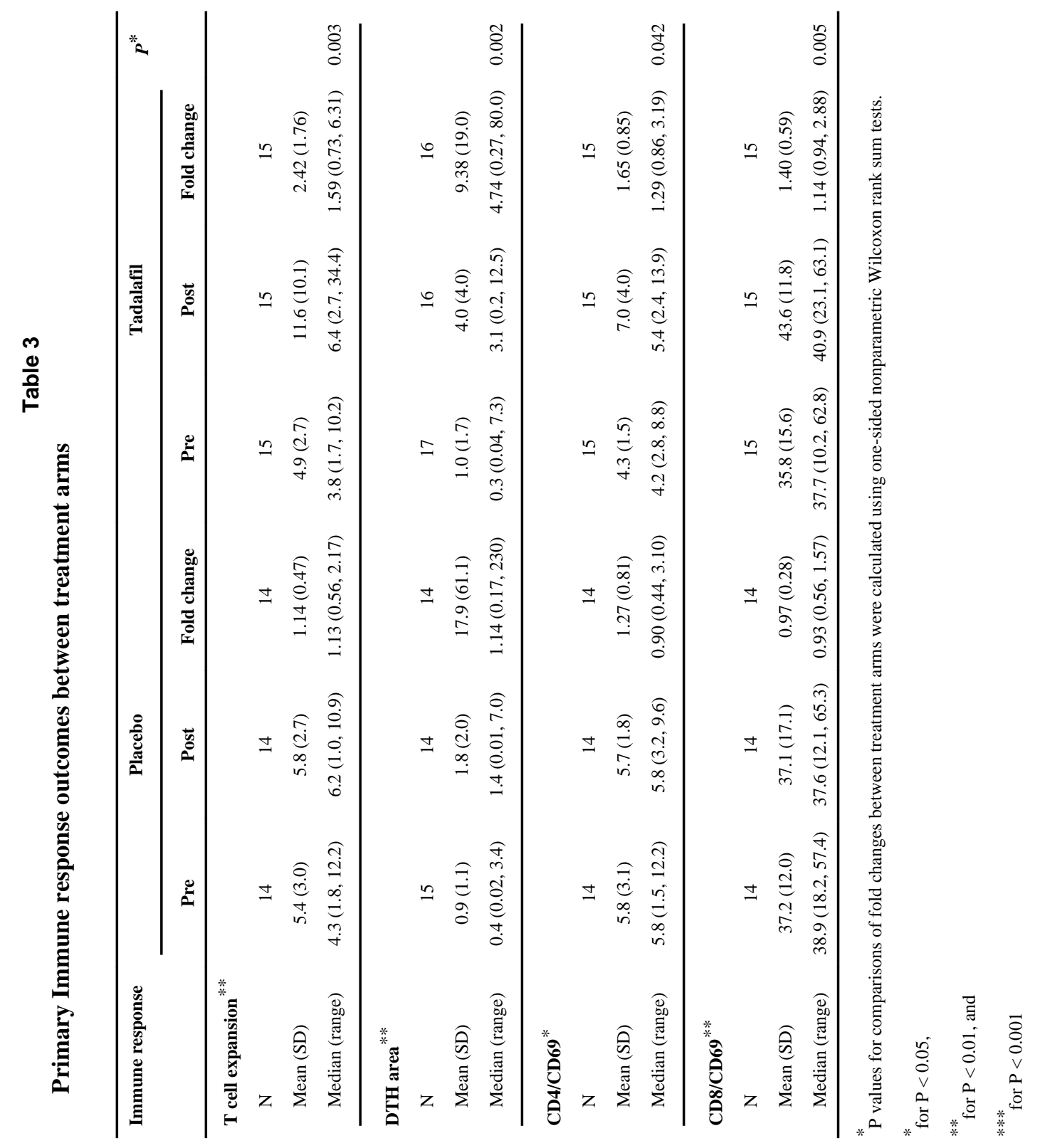

